\title{
Inhomogeneous losses and complexness of wave functions in chaotic cavities
}

\author{
D. V. Savin ${ }^{1,2}$, O. Legrand ${ }^{3}$ and F. Mortessagne ${ }^{3}$ \\ 1 Department of Mathematical Sciences, Brunel University - Uxbridge, UB8 3PH, UK \\ 2 Fachbereich Physik, Universität Duisburg-Essen - 45117 Essen, Germany \\ 3 Laboratoire de Physique de la Matière Condensée, CNRS UMR 6622 \\ Université de Nice-Sophia Antipolis - 06108 Nice cedex 2, France
}

received 2 August 2006; accepted in final form 10 October 2006

published online 1 November 2006

PACS. 05.45.Mt - Quantum chaos; semiclassical methods.

PACS. 05.60.Gg - Quantum transport.

PACS. 03.65. Nk - Scattering theory.

\begin{abstract}
In a two-dimensional microwave chaotic cavity ohmic losses located at the contour of the cavity result in different broadenings of different modes. We provide an analytic description and establish the link between such an inhomogeneous damping and the complex (non-real) character of biorthogonal wave functions. This substantiates the corresponding recent experimental findings of Barthélemy et al. [Europhys. Lett. 70 (2005) 162].
\end{abstract}

Open wave-chaotic systems in the presence of energy losses (absorption) are nowadays under intense experimental and theoretical investigations, see $[1,2]$ for recent reviews as well as [3] for a general discussion. Most of the works concern the case of uniform absorption which is responsible for homogeneous broadening $\Gamma_{\text {hom }}$ of all the modes (resonance states). However, in some experimentally relevant situations like, e.g., complex reverberant structures $[4,5]$ or even microwave cavities at room temperature $[6,7]$ one should take into account also localizedin-space losses which lead to an inhomogeneous part $\Gamma_{\text {inh }}$ of the widths which varies from mode to mode. As a result, the neighboring modes experience nontrivial correlations due to interference via one and the same decaying / dissipative environment that result in the complex-valued wave functions of corresponding resonance states. Such a complexness may reveal itself in long-range correlations of wave function intensity and current density [8] that were recently studied experimentally [9]. Following Refs. $[4,10]$, it is convenient to measure the above mentioned complexness through a single statistical parameter, namely the ratio $\left\langle(\operatorname{Im} \psi)^{2}\right\rangle /\left\langle(\operatorname{Re} \psi)^{2}\right\rangle=q^{2}$ of variances of the real and imaginary parts of the mode wave function $\psi$. The modes are real $(q=0)$ in the case of vanishing inhomogeneous losses and become complex-valued when $\Gamma_{\text {inh }} \neq 0$ (the value of $\Gamma_{\text {hom }}$ has no effect on the mode complexity, as it will become clear later on). The strong experimental evidences in the favor of the intimate relation between $q$ and $\Gamma_{\text {inh }}$ were recently provided by Barthélemy et al. [6], who analyzed hundreds of resonances in a Sinai-like chaotic microwave cavity and found a proportionality between these two quantities. Here, we present a thorough analytic description of this problem.

(c) EDP Sciences 
It is instructive to start with the discussion of the physical picture of the problematic. A typical experimental setup consists of a flat (two-dimensional) cavity fed with microwaves through attached antennas or waveguides which support $M_{a}$ scattering channels (propagating modes) in total. These very channels are used to perform all the measurements and their number is finite. Dissipation through ohmic losses at cavity boundaries gives another reason to treat our system as open, since boundaries may be viewed locally as distributed "parasitic" equivalent channels with ad hoc impedances [11,12]. One should distinguish, however, between almost uniform ohmic attenuation at the cavity plates and localized absorption at the cavity contour $[6,7]$. The number of "parasitic" channels responsible for the former ("bulk") mechanism can be naturally estimated as $M_{b} \sim(L / \lambda)^{2}$, while one has typically $M_{c} \sim L / \lambda$ channels at the contour (where $L$ is the characteristic length of the cavity and $\lambda$ denotes the wave length). Both $M_{b}, M_{c} \gg 1$ but their ratio $M_{c} / M_{b} \sim \lambda / L$ is parametrically small and that will be essential for our consideration.

It is natural, therefore, to use the following model description of the problem. According to the Hamiltonian approach to scattering [13-15], see also [2], one can represent the scattering matrix in terms of the effective non-Hermitian Hamiltonian $\mathcal{H}_{\text {eff }}$ of the open system as follows:

$$
S_{\text {tot }}=1-i V^{\dagger} \frac{1}{E-\mathcal{H}_{\mathrm{eff}}} V, \quad \mathcal{H}_{\mathrm{eff}}=H-\frac{i}{2} V V^{\dagger} .
$$

The Hamiltonian $H$ of the closed chaotic system gives rise to $N$ levels (eigenfrequencies) $\epsilon_{n}$ characterized locally in the relevant range of the energy $E$ by the mean level spacing $\Delta$. Those are coupled to all the open channels via the $N \times\left(M_{a}+M_{b}+M_{c}\right)$ matrix $V$ of coupling amplitudes and, as a result, are converted into complex resonances $\mathcal{E}_{n}=E_{n}-\frac{i}{2} \Gamma_{n}$, which are given by the poles of the $S$-matrix. Accordingly, we propose to decompose $V=\{A, B, C\}$ into coupling to $M_{a}$ antennas, $M_{b}$ "bulk" and $M_{c}$ "contour" parasitic channels to separate explicitly different contributions to the widths $\Gamma_{n}$. The total $\left(M_{a}+M_{b}+M_{c}\right)$-dimensional scattering matrix (1) is, of course, unitary $\left({ }^{1}\right)$. However, one can access experimentally only the $\left(M_{a} \times M_{a}\right)$ subblock $S=1-i A^{\dagger}\left(E-\mathcal{H}_{\mathrm{eff}}\right)^{-1} A$ which is subunitary.

Without loss of generality one can consider the case of preserved time-reversal invariance (which was indeed the case of experiment [6]) when the coupling amplitudes $V$ are real and $H$ is symmetric. It is convenient first to represent $\mathcal{H}_{\text {eff }}$ in the eigenbasis of its Hermitian part as follows:

$$
\left(\mathcal{H}_{\mathrm{eff}}\right)_{n m}=\epsilon_{n} \delta_{n m}-\frac{i}{2}\left(\sum_{a=1}^{M_{a}} A_{n}^{a} A_{m}^{a}+\sum_{b=1}^{M_{b}} B_{n}^{b} B_{m}^{b}+\sum_{c=1}^{M_{c}} C_{n}^{c} C_{m}^{c}\right) .
$$

In a chaotic cavity, $H$ is commonly described in the framework of Random Matrix Theory [3]. A (real orthogonal) rotation that diagonalizes the random Hermitian matrix $H$ transforms the (fixed) matrix $V$ to Gaussian-distributed coupling amplitudes with zero means and following covariances (we assume statistical independence of channels from different classes) [13,14]:

$$
\left\langle A_{n}^{a} A_{n^{\prime}}^{a^{\prime}}\right\rangle=2 \kappa_{a} \frac{\Delta}{\pi} \delta^{a a^{\prime}} \delta_{n n^{\prime}}, \quad\left\langle B_{n}^{b} B_{n^{\prime}}^{b^{\prime}}\right\rangle=2 \kappa_{b} \frac{\Delta}{\pi} \delta^{b b^{\prime}} \delta_{n n^{\prime}}, \quad\left\langle C_{n}^{c} C_{n^{\prime}}^{c^{\prime}}\right\rangle=2 \kappa_{c} \frac{\Delta}{\pi} \delta^{c c^{\prime}} \delta_{n n^{\prime}} .
$$

Coupling constants $\kappa$ determine transmission coefficients $T=4 \kappa /(1+\kappa)^{2}$ of the corresponding channels, so that $T \ll 1(T=1)$ stands for weak (perfect) coupling. The strong inequality $M_{b} \gg M_{c} \gg 1$ allows us to perform now the limit of a very large number of weak fictitious bulk channels, $M_{b} \rightarrow \infty$ and $T_{b} \rightarrow 0$ with $M_{b} T_{b} \equiv 2 \pi \Gamma_{\text {hom }} / \Delta$ being kept fixed [12], which singles

$\left({ }^{1}\right)$ Unitarity of the total $S$-matrix as well as causality, $\Gamma_{n}>0$, are automatically provided by the factorized algebraic structure of the anti-Hermitian part of $\mathcal{H}_{\text {eff }}[14]$. 
out the homogeneous absorption contribution. Indeed, by virtue of the central limit theorem one may replace in $\mathcal{H}_{\text {eff }}$ the sum $\sum_{b=1}^{M_{b}} B_{n}^{b} B_{m}^{b}$ with its average value $\sum_{b=1}^{M_{b}}\left\langle B_{n}^{b} B_{m}^{b}\right\rangle \equiv \Gamma_{\text {hom }} \delta_{n m}$ in the limit considered that yields

$$
\left(\mathcal{H}_{\mathrm{eff}}\right)_{n m}=\left(\epsilon_{n}-\frac{i}{2} \Gamma_{\mathrm{hom}}\right) \delta_{n m}-\frac{i}{2}\left(A A^{\mathrm{T}}+C C^{\mathrm{T}}\right)_{n m},
$$

meaning that all the levels acquire one and the same attenuation rate. Since $\mathcal{H}_{\text {eff }}$ comes into the scattering problem only as the resolvent $\left(E-\mathcal{H}_{\text {eff }}\right)^{-1}$, uniform absorption turns out to be operationally equivalent to a pure imaginary shift of the scattering energy $E \rightarrow E+\frac{i}{2} \Gamma_{\text {hom }} \equiv$ $E_{\gamma}[12,16]$. Thus the physical scattering matrix acquires the following form:

$$
S=1-i A^{\mathrm{T}} \frac{1}{E_{\gamma}-\widetilde{\mathcal{H}}_{\mathrm{eff}}} A, \quad \widetilde{\mathcal{H}}_{\mathrm{eff}}=H-\frac{i}{2}\left(A A^{\mathrm{T}}+C C^{\mathrm{T}}\right) .
$$

A representation similar to $(5)$ was used in $[5,17]$ to study statistics of transmitted power in dissipative ergodic microstructures. Here, we concentrate rather on spectroscopic problems.

It is clear from the above consideration that only escape to antennas and inhomogeneous losses contribute to the fluctuating part $\widetilde{\Gamma}_{n}=\Gamma_{n}-\Gamma_{\text {hom }}$ of the widths. The latter are given now by the imaginary parts of the complex eigenvalues of $\widetilde{\mathcal{H}}_{\text {eff. }}$. Since $\widetilde{\mathcal{H}}_{\text {eff }}$ is nonHermitian, the eigenvalue problem $\widetilde{\mathcal{H}}_{\text {eff }}|n\rangle=\widetilde{\mathcal{E}}_{n}|n\rangle$ and $\langle\tilde{n}| \widetilde{\mathcal{H}}_{\text {eff }}=\langle\tilde{n}| \widetilde{\mathcal{E}}_{n}$ defines two sets of right and left eigenfunctions, which satisfy the conditions of biorthogonality, $\langle\tilde{n} \mid m\rangle=\delta_{n m}$, and completeness, $\sum_{n=1}^{N}|n\rangle\langle\tilde{n}|=1_{N}$. The matrix $U_{n m} \equiv\langle n \mid m\rangle \neq \delta_{n m}$ differs from the unit one and is known in nuclear physics as Bell-Steinberger nonorthogonality matrix [18] (see a compact description in [14]). This matrix features in two-point correlations in open systems seen, e.g., in decay laws [19]. $U_{n n}$ appears also in optics via the so-called Petermann factor of a lasing mode [20-22]. Some statistical aspects of chaotic nonorthogonal eigenfunctions were recently studied in $[22-24]$.

For a general non-Hermitian matrix left and right eigenvectors are independent of each others. However, in our case $\widetilde{\mathcal{H}}_{\text {eff }}$ is complex symmetric (due to time-reversal invariance), implying that left eigenfunctions are given by the transpose of the right ones, $\langle\tilde{n}|=| n\rangle^{\mathrm{T}}$. As a result, $\widetilde{\mathcal{H}}_{\text {eff }}=\Psi \widetilde{\mathcal{E}} \Psi^{\mathrm{T}}$ can be diagonalized by a complex orthogonal transformation [14], with $\widetilde{\mathcal{E}}=\operatorname{diag}\left(\widetilde{\mathcal{E}}_{1}, \cdots, \widetilde{\mathcal{E}}_{N}\right)$ and $\Psi=(|1\rangle, \cdots,|N\rangle)$, that leads to the well-known pole representation of the $S$-matrix, $S=1-i A^{\mathrm{T}} \Psi\left(E_{\gamma}-\widetilde{\mathcal{E}}\right)^{-1} \Psi^{\mathrm{T}} A$, or in its components:

$$
S_{a a^{\prime}}=\delta_{a a^{\prime}}-i \sum_{n=1}^{N} \frac{\psi_{n}^{a} \psi_{n}^{a^{\prime}}}{E_{\gamma}-\widetilde{\mathcal{E}}_{n}}, \quad \psi_{n}^{a} \equiv A^{a}|n\rangle=\sum_{k=1}^{N} A_{k}^{a}|n\rangle_{k}
$$

The wave function component $\psi_{n}^{a}$ of the $n$-th mode excited through the $a$-th channel is generally complex. This complexness is solely due to biorthogonal nature of the eigenfunctions and is directly related to the structure of the anti-Hermitian part of $\widetilde{\mathcal{H}}_{\text {eff }}$. In particular, all $\psi_{n}^{a}$ would be real were $\left(\operatorname{Im} \widetilde{\mathcal{H}}_{\text {eff }}\right)_{n m} \propto \delta_{n m}$ or, more generally, if the anti-Hermitian part of the effective Hamiltonian commuted with its Hermitian part $\left({ }^{2}\right)$.

We proceed now with considering the case of tunneling coupling to antennas, which was realized in experiment [6]. This allows us to neglect safely antenna contributions to $\Gamma_{n}$, approximating $\widetilde{\mathcal{H}}_{\text {eff }} \approx H-\frac{i}{2} C C^{\mathrm{T}}$. In the case of the large but finite number $M_{c}$ of (contour)

$\left({ }^{2}\right)$ In this case both the Hermitian $H$ and anti-Hermitian parts of $\mathcal{H}_{\text {eff }}$ can be diagonalized simultaneously, thus the eigenbasis being a conventional orthogonal one, as follows from $H^{\mathrm{T}}=H$. 
channels, the levels acquire on average the width given by the so-called Weisskopf's estimate

$$
\Gamma_{\mathrm{inh}} \equiv M_{c} T_{c} \frac{\Delta}{2 \pi}
$$

well-known in nuclear physics, see, e.g., $[14,15]$. It is worth noting that this value can be formally linked to Sabine's law of room acoustics, which determines the average width $\Gamma_{\text {refl }}$ related to absorption at the cavity contour. One has $\Gamma_{\text {refl }}=c L T_{c} /(\pi S)[6,25]$, where $S$ is the cavity area, $c$ is the speed of light and $L$ is now the cavity perimeter. Making use of Weyl's law for the mean level spacing $\Delta=c \lambda / S$ and putting $M_{c}=L /(\lambda / 2)$, we find that $\Gamma_{\text {refl }}$ is exactly converted to $\Gamma_{\text {inh. }}$. This provides us with a further link between the present model description and the microscopic treatment of $[6,7]$ based on Maxwell's equations.

Fluctuations of the widths around $\Gamma_{\text {inh }}$ are mostly due to those of the matrix $C C^{\mathrm{T}}$. As follows from the central limit theorem, fluctuations of off-diagonal matrix elements are suppressed as compared to diagonal ones at $M_{c} \gg 1,\left(C C^{\mathrm{T}}\right)_{n \neq m} \sim\left(C C^{\mathrm{T}}\right)_{n n} / \sqrt{M_{c}} \sim \Gamma_{\mathrm{inh}} / \sqrt{M_{c}}$, so that they contribute to $\widetilde{\Gamma}_{n}$ in the next-to-leading order in $1 / M_{c}$. However, off-diagonal matrix elements give a dominating contribution to the mode nonorthogonality. Indeed, all essential features of the problem can be most explicitly seen in the two-state approximation. Representing $\widetilde{\mathcal{H}}_{\text {eff }}$ as follows

$$
\widetilde{\mathcal{H}}_{\mathrm{eff}} \approx\left(\begin{array}{cc}
\epsilon_{1} & 0 \\
0 & \epsilon_{2}
\end{array}\right)-\frac{i}{2}\left(\begin{array}{cc}
\left\|C_{1}\right\|^{2} & \left(C_{1}^{\mathrm{T}} C_{2}\right) \\
\left(C_{2}^{\mathrm{T}} C_{1}\right) & \left\|C_{2}\right\|^{2}
\end{array}\right)
$$

where $C_{1,2}$ are the $M_{c}$-dimensional vectors of the corresponding coupling amplitudes, one can easily solve the secular equation for its two eigenvalues $\widetilde{\mathcal{E}}_{1,2}$, finding $\widetilde{\mathcal{E}}_{1,2}=\left(\tilde{\epsilon}_{1}+\tilde{\epsilon}_{2} \mp d\right) / 2$, where $d=\sqrt{\left(\tilde{\epsilon}_{1}-\tilde{\epsilon}_{2}\right)^{2}-\left(C_{1}^{\mathrm{T}} C_{2}\right)^{2}}$ and $\tilde{\epsilon}_{1,2}=\epsilon_{1,2}-\frac{i}{2}\left\|C_{1,2}\right\|^{2}$. The corresponding eigenfunctions (we assume $\left.\epsilon_{1}<\epsilon_{2}\right)$ are given by

$$
|1\rangle=\mathcal{N}\left(\begin{array}{c}
1 \\
\text { if }
\end{array}\right) \quad \text { and } \quad|2\rangle=\mathcal{N}\left(\begin{array}{c}
-i f \\
1
\end{array}\right), \quad f=\frac{\left(C_{1}^{\mathrm{T}} C_{2}\right)}{\tilde{\epsilon}_{2}-\tilde{\epsilon}_{1}+d}
$$

$\mathcal{N}^{2}=\left(1-f^{2}\right)^{-1}$ being the normalization constant. Then the nonorthogonality matrix reads $U=|\mathcal{N}|^{2}\left[\left(1+|f|^{2}\right) 1_{2}+2 \operatorname{Re}(f) \sigma_{y}\right]$, with the Pauli matrix $\sigma_{y}$. The parameter $f$ controls the mode complexness, as follows from

$$
\psi_{1,2}^{a}=\mathcal{N}\left(A_{1,2}^{a} \pm \text { if } A_{2,1}^{a}\right)
$$

for the mode wave functions.

In experiment [6], the complexness parameter

$$
q^{2}=\left\langle\left(\operatorname{Im} \psi_{n}^{a}\right)^{2}\right\rangle /\left\langle\left(\operatorname{Re} \psi_{n}^{a}\right)^{2}\right\rangle
$$

was accessible only in the regime of the weak mode overlap due to inhomogeneous losses (we stress, however, that the total width $\Gamma_{\text {hom }}+\Gamma_{\text {inh }}$ can be larger than $\Delta$ ). In this regime, $\Gamma_{\text {inh }} \ll$ $\Delta$, the computation of $q$ can be easily carried out by making the use of the above two-state approximation. One finds straightforwardly that $q^{2} \approx\left\langle(\operatorname{Re} f)^{2}\right\rangle \approx\left\langle\left(C_{1}^{\mathrm{T}} C_{2}\right)^{2}\right\rangle /(2 \Delta)^{2}$, which determines now the average nonorhogonality matrix as $\left\langle U_{n m}\right\rangle \approx\left(1+2 q^{2}\right) \delta_{n m}$. The remaining Gaussian averaging over random amplitudes (3) yields $\sum_{c, c^{\prime}}\left\langle C_{1}^{c} C_{2}^{c} C_{1}^{c^{\prime}} C_{2}^{c^{\prime}}\right\rangle=M_{c}\left(2 \kappa_{c} \Delta / \pi\right)^{2} \approx$ $M_{c}\left(T_{c} \Delta / 2 \pi\right)^{2}$, as $T_{c} \approx 4 \kappa_{c} \ll 1$ in our case of large $M_{c}$ and fixed $\Gamma_{\text {inh. }}$. Collecting everything, we arrive at

$$
q \approx \frac{1}{\sqrt{M_{c}}} \frac{\Gamma_{\mathrm{inh}}}{2 \Delta}
$$


that substantiates the proportionality between $q$ and $\Gamma_{\text {inh }}$ established experimentally [6]. The mode complexness (nonorthogonality) decreases as the number of absorptive channels increases, in agreement with the general discussion presented above.

We discuss now the role of fluctuations in energy levels and contributions from the other levels neglected so far. Restricting ourselves to the same weak overlap regime, we can use the perturbation theory for wave functions to find $q^{2} \approx \frac{1}{4} \sum_{m \neq n}\left\langle\left(C_{n}^{\mathrm{T}} C_{m}\right)^{2}\right\rangle\left\langle\left(\epsilon_{n}-\epsilon_{m}\right)^{-2}\right\rangle$. The known correlation function $R_{2}(\omega)$ of Gaussian orthogonal ensembles, see [3], can be now used to get $\sum_{m \neq n}\left\langle\left(\epsilon_{n}-\epsilon_{m}\right)^{-2}\right\rangle=\int d \omega \omega^{-2} R_{2}(\omega)$, which can be appreciated as (average square of) the so-called "level curvature" studied in [26,27]. Since $R_{2}(\omega) \sim \omega$ as $\omega \rightarrow 0$, this integral has a logarithmic divergency regularized by setting the lower limit $\sim \Gamma_{\text {inh }}$ that also ensures us to stay within the perturbation theory. As a result, (12) is renormalized to yield a contribution $\sim \ln \left(\Delta / \Gamma_{\mathrm{inh}}\right)$. One should expect that such a factor would be absent in a non-perturbative treatment (see also the relevant discussion in [22]). The computation of $q$ at arbitrary inhomogeneous absorption is still an interesting open problem to consider.

In summary, we have presented the model description for inhomogeneous (localized-inspace) losses in open chaotic systems and discussed thoroughly the resulting complexness (or biorthogonality) of the mode wave functions. In particular, the complexness parameter $q$ determining the relative weight of the imaginary parts of the modes is found analytically to be proportional to the inhomogeneous part $\Gamma_{\text {inh }}$ of the widths in full agreement with experimental results of [6]. Though the present calculation of $q$ is perturbative in the small parameter $\Gamma_{\text {inh }} / \Delta$, it may nevertheless be valid in the intermediate or large modal overlap regime where $\Gamma_{\text {hom }}$ dominates, as, for instance, in the case of room acoustics or elastodynamics [1,25]. Our analysis may be also relevant for problems of mode nonorthogonality outside of the scattering systems as those considered recently in $[23,28]$.

$$
* * *
$$

We thank Y. V. Fyodorov and V. V. Sokolov for useful comments. One of us (DVS) acknowledges gratefully the generous hospitality of LPMC in Nice and the financial support of University of Nice during his stay there. The financial support by the SFB/TR 12 of the DFG (DVS) is acknowledged with thanks.

\section{REFERENCES}

[1] Kuhl U., Stöckmann H.-J., and Weaver R., J. Phys. A: Math. Gen., 38 (2005) 10433.

[2] Fyodorov Y. V., Savin D. V., and Sommers H.-J., J. Phys. A: Math. Gen., 38 (2005) 10731.

[3] Stöckmann H.-J., Quantum Chaos: An Introduction (Cambridge University Press, Cambridge, UK) 1999.

[4] Lobkis O. I. and Weaver R. L., J. Acoust. Soc. Am., 108 (2000) 1480.

[5] Rozhkov I., Fyodorov Y. V., and Weaver R. L., Phys. Rev. E, 68 (2003) 016204.

[6] Barthélemy J., Legrand O., and Mortessagne F., Europhys. Lett., 70 (2005) 162.

[7] Barthélemy J., Legrand O., and Mortessagne F., Phys. Rev. E, 71 (2005) 016205.

[8] Brouwer P. W., Phys. Rev. E, 68 (2003) 046205.

[9] Kim Y.-H., Kuhl U., Stöckmann H.-J., and Brouwer P. W., Phys. Rev. Lett., 94 (2005) 036804.

[10] Pnini R. and Shapiro B., Phys. Rev. E, 54 (1996) R1032.

[11] Lewenkopf C. H., Müller A., and Doron E., Phys. Rev. A, 45 (1992) 2635.

[12] Brouwer P. W. and Beenakker C. W. J., Phys. Rev. B, 55 (1997) 4695.

[13] Verbaarschot J. J. M., Weidenmüller H. A., and Zirnbauer M. R., Phys. Rep., 129 (1985) 367. 
[14] Sokolov V. V. and Zelevinsky V. G., Nucl. Phys. A, 504 (1989) 562.

[15] Fyodorov Y. V. and Sommers H.-J., J. Math. Phys., 38 (1997) 1918.

[16] Savin D. V. and Sommers H.-J., Phys. Rev. E, 68 (2003) 036211.

[17] Rozhrov I., Fyodorov Y. V., and Weaver R. L., Phys. Rev. E, 69 (2004) 036206.

[18] Bell J. S. and Steinberger J., Proceedings of the Oxford International Conference on Elementary Particles, September, 1965 (Rutherford High Energy Laboratory, Chilton, Berkshire, UK) 1966, p. 195.

[19] Savin D. V. and Sokolov V. V., Phys. Rev. E, 56 (1997) R4911.

[20] Petermann K., IEEE J. Quant. Electron., 15 (1979) 566.

[21] Siegman A. E., Phys. Rev. A, 39 (1989) 1253.

[22] Schomerus H., Frahm K. M., Patra M., and Beenakker C. W. J., Physica A, 278 (2000) 469.

[23] Chalker J. T. and Mehlig B., Phys. Rev. Lett., 81 (1998) 3367.

[24] Fyodorov Y. V. and Menlig B., Phys. Rev. E, 66 (2002) 045202(R).

[25] Mortessagne F., Legrand O., and Sornette D., Chaos, 3 (1993) 529.

[26] Fyodorov Y. V., and Sommers H.-J., Z. Phys. B, 99 (1995) 123.

[27] von Oppen F., Phys. Rev. E, 51 (1995) 2647.

[28] Fyodorov Y. V. and Sommers H.-J., J. Phys. A: Math. Gen., 36 (2003) 3303. 